\title{
Inventario de Conductas Infantiles en preescolares: Propiedades psicométricas del Inventario de Conductas Infantiles (CBCL/1.5-5) y del Informe del Cuidador/Educador (C-TRF) en Preescolares Chilenos
}

\section{Psychometric Properties of the Child Behavior Checklist and the Caregiver/Teacher Report Form in Chilean Preschoolers}

\author{
Félix Cova \\ Claudio Bustos \\ Paulina Rincón \\ Departamento de Psicología, Universidad de Concepción, Concepción, Chile. \\ Sandra Saldivia \\ Carolina Inostroza \\ Departamento de Psiquiatría y Salud Mental, Universidad de Concepción. Concepción, Chile. \\ Pamela Grandón \\ Departamento de Psicología, Universidad de Concepción, Concepción, Chile. \\ Rec. (05 de abril de 2016) Acept. (11 de noviembre de 2016)

\begin{abstract}
Resumen
El Inventario de Conductas Infantiles 1.5-5 años (CBCL /1.5-5 por su acrónimo en inglés) y el Informe del Cuidador/Educador (C-TRF por su acrónimo en inglés), son de los instrumentos más ampliamente reconocidos internacionalmente para evaluar conductas desadaptativas que pueden afectan el desarrollo presente y futuro de niños y niñas preescolares. Se evaluó el comportamiento psicométrico de ambos instrumentos en una muestra diversa socioeconómicamente de 550 preescolares chilenos. En general, ambos mostraron un comportamiento psicométrico análogo al obtenido con estudios en otras sociedades. Los análisis factoriales confirmatorios mostraron un ajuste adecuado del modelo bifactorial original. Sin embargo, en el CBCL /1.5-5 los puntajes obtenidos son más altos que en la mayoría de los países y, comparativamente, en ambos instrumentos, se observó un mayor efecto de género. Se analizan los hallazgos obtenidos, y se dan sugerencias normativas y conceptuales para el empleo de ambos instrumentos en niños y niñas preescolares en nuestro país.

Key words: comportamientos externalizados e internalizados-AFC-preescolares-evaluación.
\end{abstract}

\begin{abstract}
The Child Behavior Checklist/1.5-5 and the Caregiver /Teacher Report Form are one of the most widely internationally recognized instruments to evaluate desadaptative behaviors which may affect the current and future development of preschool children. The psychometric behavior of both instruments was evaluated in a socioeconomically diverse sample of 550 Chilean preschool children. In general, it was observed an analog behavior of both compared to the one obtained with studies in other societies. The confirmatory factor analysis showed a suitable adjustment of the original bifactor model. However, in the CBCL, the scores obtained are higher than in most of the countries and, comparatively, in both instruments, a greater effect of gender was observed. The findings obtained are analyzed and, normative and conceptual suggestions are given for the use of both instruments in preschool children in our country.

Key words: internalizing and externalizing behaviors-CFA-preschools children-assesment.
\end{abstract}

\footnotetext{
Correspondencia: Félix Cova Solar fecova@udec.cl. Dirección Postal: Las Margaritas 1455 Ed. Parque 407, Huertos

${ }^{A}$ gradecimientos: Este estudio fue financiado a través del proyecto FONDECYT 11200716.
} 


\section{Introducción}

Problemas emocionales (conductas internalizadas) y conductuales (conductas externalizadas) en la niñez temprana, (0-6 años) pueden afectar el bienestar presente y futuro de niños y niñas (Egger y Angold, 2006; Rosseel, 2012). Estos problemas, en determinados contextos, tienen el riesgo de estabilizarse o agravarse posteriormente. Se ha observado que conductas internalizadas o externalizadas en niños y niñas pequeños son un factor de riesgo de diversas dificultades psicopatológicas en la adolescencia y adultez. Las conductas externalizadas son las que han mostrado mayor riesgo de continuidad futura en diversas manifestaciones (Hofstra, van der Ende y Verhulst, 2002) .

De allí la relevancia de la intervención temprana en relación a estas conductas, en términos preventivos o terapéuticos, y la necesidad de contar con instrumentos que faciliten la evaluación de estas conductas. Los cuestionarios destinados a recoger las impresiones que tienen distintos informantes que conocen a los niños y niñas respecto de la presencia y frecuencia de distintos comportamientos, son uno de los instrumentos más útiles para ayudar a esas evaluaciones (Rescorla et al., 2014). Además de ser de relativamente fácil aplicación, tienen el valor de ser medidas con validez ecológica, que permiten tener una aproximación al comportamiento habitual que el niño o niña tiene en distintos contextos.

Entre los cuestionarios que han alcanzado mayor popularidad en la evaluación del comportamiento de niños y niñas están los que componen el Achenbach System of Empirically Based Assessment ASEBA, desarrollado en trabajo de décadas por Thomas Achenbach y su equipo. ASEBA tiene versiones diferentes para distintos grupos etarios. En específico, entre los más estudiados y utilizados, están la Child Behavior Cheklist CBCL, aplicados a los cuidadores primarios (madres, padres), y el Teacher Repor Form TRF, aplicado a los educadores. Estos cuestionarios están destinados a evaluar la presencia de las conductas más habituales que generan preocupación respecto de niños y niñas, han sido estudiados en diversas partes del mundo, en varios idiomas, mostrando propiedades psicométricas sólidas. Pese a su relevancia, la versión correspondiente a niñas y niños pequeños o preescolares ha sido menos estudiada comparativamente, aunque existe creciente interés (Ivanova et al., 2010; Lecannelier et al., 2014; Liu, Cheng,y Leung, 2011; Rescorla et al., 2012) . La versión más actualizada de estos instrumentos fue publicada por Achenbach y Rescorla el año 2000, y considera el rango etáreo 1.5 a 5 años. Fueron denominados CBCL/1.5-5 y C-TRF, respectivamente
(Achenbach y Rescorla, 2000). Los puntajes de la CBCL/1.5 y C-TRF muestran capacidad para discriminar entre niños y niñas referidos y no referidos a servicios de salud mental y son predictores de dificultades posteriores en el desarrollo infantil (Achenbachy Rescorla, 2000).

$\mathrm{Al}$ igual que en la CBCL y TRF para niños y niñas mayores a 6 años, en la CBCL/1.5-5 y C-TRF, se ha identificado un modelo de estructura factorial de los instrumentos de dos niveles. Este modelo es resultado de los análisis factoriales realizados por los autores de los cuestionarios en muestras norteamericanas. El primer nivel corresponde a los siguientes 6 factores de primer orden comunes a ambos instrumentos: reactividad emocional, problemas ansiodepresivos, quejas somáticas, retraimiento, problemas atencionales y agresividad. En la CBLC/1.5-5 se distingue además, otro factor de primer orden: problemas del sueño (por ello, la CBCL/1.5-5 tiene 7 factores de primer orden). El segundo nivel, también en ambos instrumentos, es bifactorial, identificándose un factor de segundo orden de comportamientos internalizados (que incluye problemas ansiodepresivos, quejas somáticas, retraimiento) y otro factor de segundo orden de comportamientos externalizados (que incluye problemas atencionales y agresividad). Problemas del sueño no se relacionan con ningún factor de segundo orden, por lo que, en ambos instrumentos, el modelo de segundo orden incluye los mismos 6 factores de primer orden. Para su uso clínico y en investigación, es posible emplear los factores de primer y segundo orden como escalas, así como el puntaje total. También para ambos instrumentos se han derivado síndromes analogables a los criterios diagnósticos del DSM-IV pero ello no será analizado en este artículo.

La utilidad y validez de la distinción entre comportamientos internalizados y externalizados, es un hallazgo recurrente de las investigaciones, y presentan importantes grados de relación entre sí (Mesman, Bongers,y Koot, 2001). Menos claridad existe respecto de los comportamientos específicos que son englobables en cada una de estas dimensiones, y respecto de los cambios configuracionales de estas dimensiones a lo largo de la vida. En específico, en el caso de la CBCL/1.5-5 y C-TRF, por ejemplo, los problemas atencionales son parte del factor de segundo orden de comportamientos externalizados, pero en niños y niñas mayores, se le identifica como un factor independiente de los comportamientos externalizados.

Existen algunos estudios independientes (Lecannelier et al., 2014; Tan, Dedrick,y Marfo, 2007) y otros realizados por los mismos autores de los cuestionarios en asociación a diversos equipos de investigación multiculturales (Ivanova et al., 2010), que han permitido contrastar este modelo 
bifactorial de la CBCL/1.5-5 y C-TRF en diferentes sociedades. En los análisis multiculturales de la CBCL/1.5-5 realizados por Achenbach y su equipo, el modelo alcanzó un buen ajuste en las 23 sociedades estudiadas, si bien en 9 la matriz de covarianza no fue positivamente definida; la correlación del factor reactividad emocional y ansiosodepresivo fue muy elevada en varias sociedades, aunque no se observó que combinar esos factores mejorara el ajuste. El ajuste del modelo para C-TRF fue evaluado en muestras de 14 sociedades y mostró un ajuste aceptable pero, en la mayoría la matriz de covarianza, no fue positivamente definida. La combinación del factor reactividad emocional y ansiosodepresivo resolvió este problema en 8 sociedades (Ivanova et al., 2011).

Del CBCL/1.5-5 recientemente se ha publicado un análisis confirmatorio de su estructura factorial en una muestra de niños y niñas chilenos/as. La estructura de primer orden ajustó, en general, en forma adecuada (excepto problemas del sueño). El análisis de la estructura bifactorial de segundo orden (se hizo considerando "parcels" y no el conjunto de ítems de los factores de primer orden) también ajustó adecuadamente (Lecannelier et al., 2014). En Chile, la C-TRF fue estudiada como parte del estudio multicultural citado; el modelo original no ajustó; sí se observó ajuste de un modelo de un solo nivel que distingue comportamientos internalizados y externalizados (Ivanova et al., 2011).

Achenbach et al. (Achenbach y Rescorla, 2010) distinguen tres agrupaciones de sociedades en función del nivel de puntajes en la escala total del CBCL/1.5-5 y C-TRF obtenidos en los estudios realizados en éstas. Luego de obtener un promedio "omnibús" de los puntajes en las distintas sociedades, distinguen un grupo 1 con puntajes una DT por debajo del promedio, un grupo 3 con puntajes una DT por sobre el promedio, y un grupo 2 con puntajes dentro del rango del promedio. El promedio omnibús para el CBCL/1.5-5 fue de 32.8 (DT=7.0) y de C-TRF fue de 23.4 $(\mathrm{DT}=8.6)$. En general, observan pocos efectos de género y edad en ambos instrumentos, existiendo una tendencia de los niños a tener puntajes más altos que las niñas en algunas escalas; también existe una tendencia a que se observen más conductas desadaptativas en niños y niñas de menor edad que en los mayores.

El presente estudio tuvo el propósito de evaluar el comportamiento de la CBCL/1.5-5 y C-TRF en preescolares chilenos. Se analiza la estructura factorial, se comparan los puntajes con los obtenidos en otros contextos y se analizan las diferencias de género y edad. Se indican asimismo los promedios de puntajes de las escalas más relevantes, en centiles de relevancia, para facilitar el uso de estos instrumentos en nuestro país.

\section{Método}

\section{Participantes}

El estudio incluyó 550 participantes: 284 niños (52\%) y 266 (48\%) niñas de 2 a 5 años inclusive ( $M=3, D S=0.93)$. La muestra era diversa socioeconómicamente. Como indicador indirecto del nivel socioeconómico se puede considerar el tipo de institución educativa a que asistían los niños y niñas: 93 participantes $(17 \%)$ asistían a instituciones privadas pagadas (NSE alto y medio alto), 97(18\%) a instituciones particular subvencionadas (NSE medio y bajo) y 360 (65\%), a instituciones públicas o fundaciones focalizadas en población económicamente vulnerable (NSE bajo). Los instrumentos fueron a aplicados a las figuras parentales de los niños y niñas (madres, en su mayoría, 490, 89\% del total) y a sus educadoras.

\section{Instrumentos}

Child Behavior Checklist/ 11/2-5 (CBCL/ 11/2-5) y Teacher Report Form, (C-TRF) (Achenbach y Rescorla, 2000).

CBCL/ 1 1 $\frac{1}{2-5}$ y C-TRF son instrumentos de 100 ítems cada uno con tres opciones de respuesta tipo Likert, que evalúan la presencia de conductas desadaptativas en niños y niñas preescolares. El CBCL $1 \frac{1}{2}$ es respondido por la madre, padre o figura parental y el C-TRF, por personal educativo a cargo del niño o niña que tenga contacto frecuente con él/ella. Está traducido a diversos idiomas, incluyendo el castellano. Sus propiedades psicométricas han sido corroboradas en diversas investigaciones, incluyendo estudios en Chile (Lecannelier et al., 2014).

\section{Resultados}

Se realizó un análisis factorial confirmatorio, usando el paquete lavaan de R (Rosseel, 2012). Se utilizó estimación WLSMV, considerando los ítems como variables categóricas. Como indicadores de bondad se utilizó el Chi-cuadrado ajustado de Satorra-Bentler, robusto a la falta de normalidad de los datos, así como CFI, TLI y RMSEA, todos ajustados. 
Inicialmente, se evaluó el ajuste de los modelos originales de la CBCL/1.5-5 y C.-TRF. Para ello, se probó el modelo de primer orden, con 7 factores para CBCL y de 6 factores para CTRF; ambos modelos mostraron un ajuste adecuado (Tabla 1). Todos las cargas de los ítems, en los factores de primer orden, fueron estadísticamente significativas $(\mathrm{p}<.01)$ para la $\mathrm{CBCL} / 1.5-5$, con una mediana de $.60 \mathrm{y}$ un rango .20-.79. En el caso de C-TRF, todos los ítems fueron también estadísticamente significativos $(\mathrm{p}<.01)$, excepto el ítem 86 en el factor 3 (quejas somáticas); la mediana de las cargas factoriales fue de .74 y el rango estuvo entre .07-.99.

Luego se probó el modelo bifactorial de segundo orden, con 6 factores de primer orden. Para ambos instrumentos fue necesario realizar una búsqueda de especificación, ya que este modelo, si bien tenía un adecuado ajuste (RMSEA de 029 y .047 para CBCL y CTRF, respectivamente), presentaba parámetros fuera de límites, producto de una correlación superior a 1.0 entre el factor de reactividad emocional con el factor de segundo orden de internalización. Como opción de solución de este problema, para ambos instrumentos, se restringió la varianza residual del factor de reactividad emocional, de modo que fuese idéntica a la del factor ansioso/depresivo que también presentaba una relación muy alta con internalización.

Para la CBCL/1.5-5 el modelo resultante mostró un ajuste adecuado (RMSEA .029, ver Tabla 1) sin presentar parámetros fuera de rango. La figura 1 muestra las cargas factoriales del modelo. La correlación latente entre el factor internalizado y externalizado fue de .80. Las cargas de los factores de primer orden fueron todas estadísticamente significativas y superiores a .70. En particular, las cargas de los factores emocionalmente reactivo y ansiosodepresivo fueron muy altas, .99 y .98 respectivamente. Para la C-TRF el modelo resultante también mostró un ajuste adecuado (RMSEA
.048), sin parámetros fuera de rango (figura 2). La correlación latente entre el factor internalizado y externalizado fue de .89. Las cargas de los factores de primer orden fueron todas estadísticamente significativas y superiores a .70 .

El alfa de Cronbach en el CBCL/1.5-5 fue superior a .6 en todos los factores de primer orden, excepto quejas somáticas (.54) y problemas de atención (.51), y superior a .84 en los factores de segundo orden y puntaje total. En el C-TRF sólo quejas somáticas tuvo un alfa inferior a .60; los factores de segundo orden y puntaje total mostraron valores superiores a .86 .

El promedio total de conductas desadaptativas, de acuerdo al CBCL/1.5-5, fue de 45.52 (DT=21.96), de conductas internalizadas de $12.8(\mathrm{DT}=8.22)$ y de conductas externalizadas de 16.89 (8.26). El promedio total de conductas desadaptativas, de acuerdo al C-TRF, fue de 28.94 ( $\mathrm{DT}=22.80$ ), de conductas internalizadas de $7.65(\mathrm{DT}=6.76)$ y de conductas

Figura 1. Modelo factorial para $C B C L$

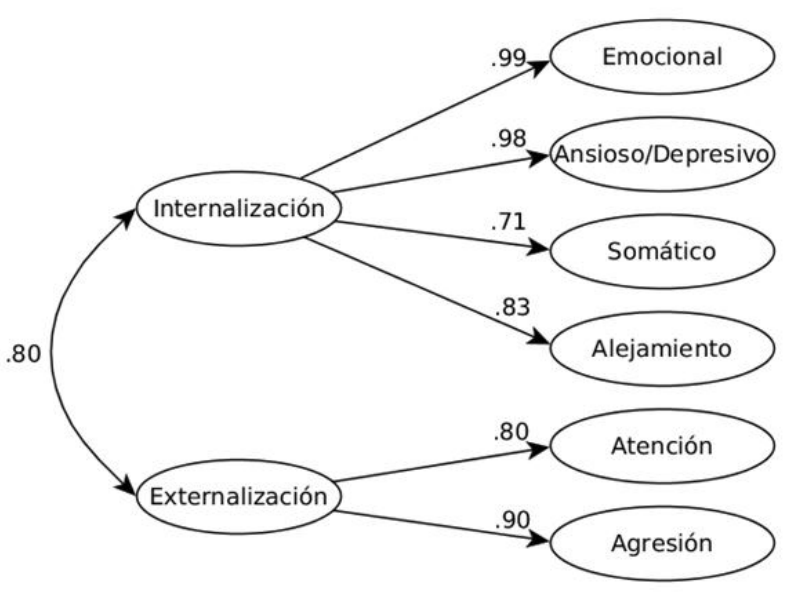

Tabla 1. Índices de ajuste de análisis factorial confirmatorio CBCL/1.5-5 y C-TRF

\begin{tabular}{|c|c|c|c|c|c|}
\hline Modelo & $\mathrm{X}^{2}$ & $\mathrm{X}^{2} / \mathrm{gl}$ & CFI & TLI & RMSEA \\
\hline CBCL: Modelo de 7 factores primer orden & $\mathrm{X}^{2}(2123)=2889.98, \mathrm{p}<0.001$ & 1.361 & 0.907 & 0.903 & $\begin{array}{l}0.026[0.023, \\
0.028], \mathrm{p}=1.000\end{array}$ \\
\hline $\begin{array}{l}\text { CBCL: Modelo bifactorial segundo orden ( } 6 \\
\text { factores primer orden y } 2 \text { segundo orden), } \\
\text { con factores } 1 \text { y } 2 \text { de segundo orden con } \\
\text { cargas y varianzas iguales }\end{array}$ & $X^{2}(1705)=2465.86, p<0.001$ & 1.446 & 0.901 & 0.898 & $\begin{array}{l}0.029[0.026 \\
0.031], p=1.000\end{array}$ \\
\hline C-TRF: Modelo de 6 factores primer orden & $\mathrm{X}^{2}(2064)=4455.87, \mathrm{p}<0.001$ & 2.159 & 0.892 & 0.887 & $\begin{array}{l}0.046[0.044 \\
0.048], p=1.000\end{array}$ \\
\hline $\begin{array}{l}\text { C-TRF: Modelo bifactorial segundo orden } \\
\text { ( } 6 \text { factores primer orden y } 2 \text { segundo orden), } \\
\text { con factores } 1 \text { y } 2 \text { de segundo orden con } \\
\text { cargas y varianzas iguales }\end{array}$ & $X^{2}(2074)=4653.24, p<0.001$ & 2.244 & 0.88 & 0.876 & $\begin{array}{l}0.048[0.046 \\
0.049], p=0.988\end{array}$ \\
\hline
\end{tabular}


Figura 2. Modelo factorial para C-TRF

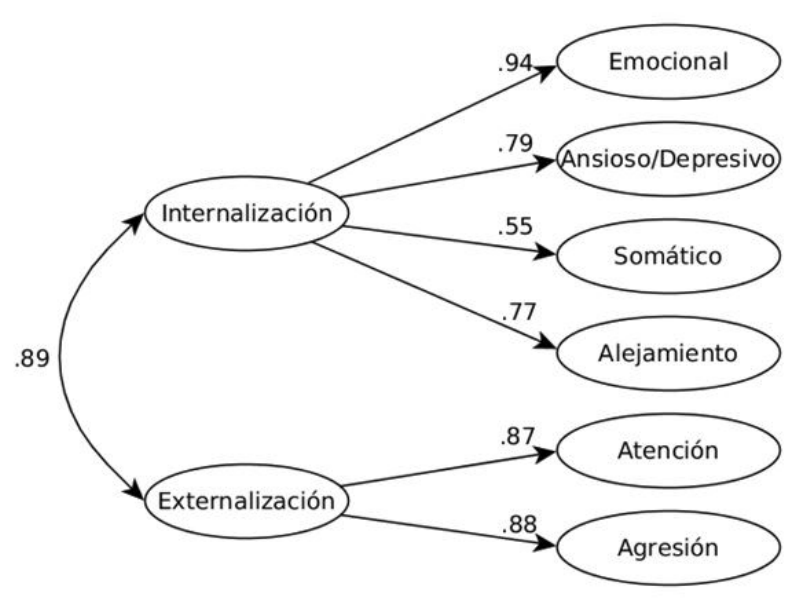

externalizadas de 12.16 (12.06). En la tablas 2 y 3 se muestran los promedios de las escalas diferenciados por sexo.

No se observó un patrón consistente de diferencias por edad en las diferentes escalas en la $\mathrm{CBCL} / 1.5-5$, aunque se observó una tendencia a que se observaran más conductas desadaptativas a los tres años. Lo propio ocurrió en la C-TRF, aunque aquí los valores más altos se observaron a los dos años. Sin embargo, al comparar niños y niñas de 3 y 2 años, respectivamente, con niños y niñas de 5 años en la $\mathrm{CBCL} / 1.5-5$ y C-TRF, solo se observaron diferencias estadísticamente significativas $(\mathrm{p}<.05)$ en conductas internalizadas en la CBCL/1.5-5 (promedio a los 3 años de 13.5 $\mathrm{DT}=8.26$ y a los 5 de $12.7 \mathrm{DT}=7.6$ ), en aislamiento en la C-TRF (promedio a los 2 años de $3.70 \mathrm{DT}=3.59$ y a los 5 de $2.48 \mathrm{DT}=3.29$ ) y en conductas agresivas (promedio a los 2 años de $11.17 \mathrm{DT}=9.54$ y a los 5 de $7.74 \mathrm{DT}=9.33$ ).

Tabla 2. Promedio CBCL/1.5-5 en niñas y niños

\begin{tabular}{lccccccc}
\hline \multicolumn{1}{c}{ Escalas } & \multicolumn{2}{c}{ Niñas } & \multicolumn{2}{c}{ Niños } & U Mann-Whitney & valor.p & ES(d) \\
\hline & M & DT & M & DT & & & \\
\hline Reactividad emocional & 2.905 & 2.663 & 3.214 & 2.897 & 34264.50 & 0.257 & 0.11 \\
Ansiosodepresivo & 4.232 & 2.684 & 4.496 & 2.901 & 34708.50 & 0.3775 & 0.09 \\
Quejas somáticas & 2.852 & 2.345 & 3.047 & 2.368 & 34244.00 & 0.2519 & 0.08 \\
Aislamiento & 2.312 & 2.259 & 2.511 & 2.314 & 34133.00 & 0.2241 & 0.09 \\
Problemas para dormer & 2.992 & 2.67 & 3.29 & 2.462 & 32812.50 & 0.05195 & 0.12 \\
Problemas de atención & 2.878 & 1.818 & 3.46 & 1.802 & 29640.50 & 0.0001941 & 0.32 \\
Conductas agresivas & 13.15 & 6.799 & 14.26 & 7.307 & 33177.50 & 0.08439 & 0.16 \\
Conductas internalizadas & 12.3 & 8.009 & 13.27 & 8.395 & 33866.00 & 0.1788 & 0.12 \\
Conductas externalizadas & 16.03 & 7.993 & 17.72 & 8.435 & 31981.00 & 0.01694 & 0.21 \\
Puntaje total & 43.29 & 21.07 & 47.64 & 22.6 & 32387.00 & 0.03064 & 0.2 \\
\hline
\end{tabular}

Tabla 3. Promedio por sexo C-TRF

\begin{tabular}{lccccccc}
\hline \multicolumn{1}{c}{ Escalas } & \multicolumn{2}{c}{ Niñas } & \multicolumn{2}{c}{ Niños } & U Mann-Whitney & valor.p & ES(d) \\
\hline Reactividad emocional & 1.286 & 1.798 & 1.434 & 1.91 & $\mathrm{U}=36647.50$ & 0.4313 & 0.08 \\
Ansiosodepresivo & 2.421 & 2.333 & 2.654 & 2.474 & $\mathrm{U}=36129.50$ & 0.3015 & 0.1 \\
Quejas somáticas & 0.9549 & 1.45 & 0.8881 & 1.285 & $\mathrm{U}=38550.50$ & 0.7648 & 0.05 \\
Aislamiento & 2.335 & 2.866 & 3.29 & 3.568 & $\mathrm{U}=32539.50$ & 0.00278 & 0.29 \\
Problemas de atención & 3.169 & 3.07 & 5.108 & 4.242 & $\mathrm{U}=28158.00$ & $1.052 \mathrm{e}-07$ & 0.52 \\
Conductas agresivas & 5.853 & 6.929 & 9.962 & 10.33 & $\mathrm{U}=28554.50$ & $3.708 \mathrm{e}-07$ & 0.46 \\
Conductas internalizadas & 6.996 & 6.237 & 8.266 & 7.163 & $\mathrm{U}=34516.50$ & 0.0595 & 0.19 \\
Conductas externalizadas & 9.023 & 9.142 & 15.07 & 13.64 & $\mathrm{U}=27842.50$ & $4.998 \mathrm{e}-08$ & 0.52 \\
Puntaje total & 24.05 & 18.83 & 33.49 & 25.14 & $\mathrm{U}=29372.50$ & $3.675 \mathrm{e}-06$ & 0.42 \\
\hline
\end{tabular}


Tabla 4. Puntajes centiles seleccionados CBCL/1.5-2

\begin{tabular}{ccccccc}
\hline & \multicolumn{2}{c}{ Niñas } & \multicolumn{3}{c}{ Niños } \\
\hline & Total & $\begin{array}{c}\text { Conductas } \\
\text { internalizadas }\end{array}$ & $\begin{array}{c}\text { Conductas } \\
\text { Externalizadas }\end{array}$ & Total & $\begin{array}{c}\text { Conductas } \\
\text { internalizadas }\end{array}$ & $\begin{array}{c}\text { Conductas } \\
\text { Externalizadas }\end{array}$ \\
\hline $50 \%$ & 41 & 11 & 15 & 45 & 12 & 17 \\
$60 \%$ & 45 & 12 & 17 & 51 & 14 & 19 \\
$70 \%$ & 52 & 15 & 20 & 60 & 16 & 23 \\
$80 \%$ & 60 & 19 & 22 & 67 & 19 & 25 \\
$83 \%$ a & 63 & 19 & 23 & 70 & 22 & 30 \\
$90 \%$ & 72 & 22 & 26 & 78 & 26 & 32 \\
$95 \%$ & 83 & 29 & 32 & 86 & 30 & 25 \\
\hline
\end{tabular}

Tabla 5. Puntajes centiles seleccionados C-TRF

\begin{tabular}{ccccccc}
\hline & \multicolumn{2}{c}{ Niñas } & \multicolumn{2}{c}{ Niños } \\
\hline & Total & $\begin{array}{c}\text { Conductas } \\
\text { internalizadas }\end{array}$ & $\begin{array}{c}\text { Conductas } \\
\text { Externalizadas }\end{array}$ & Total & $\begin{array}{c}\text { Conductas } \\
\text { internalizadas }\end{array}$ & $\begin{array}{c}\text { Conductas } \\
\text { Externalizadas }\end{array}$ \\
\hline $\mathbf{5 0 \%}$ & 19 & 6 & 6 & 28 & 6 & 12 \\
$\mathbf{6 0 \%}$ & 24 & 7 & 9 & 34 & 8 & 15 \\
$\mathbf{7 0 \%}$ & 30 & 9 & 12 & 42 & 10 & 20 \\
$\mathbf{8 0 \%}$ & 36 & 11 & 16 & 50 & 14 & 25 \\
$\mathbf{8 3 \%}$ & 40 & 12 & 18 & 55 & 15 & 26 \\
$\mathbf{9 0 \%}$ & 51 & 16 & 21 & 68 & 18 & 34 \\
\hline
\end{tabular}

Las tablas 4 y 5 indican los puntajes de niños en niños en algunos centiles relevantes.

\section{Discusión}

Dadas las implicaciones de los problemas de salud mental en la niñez temprana, es de relevancia poder contar con instrumentos que faciliten la evaluación de estas conductas, ojalá de uso sencillo, y que recojan las evaluaciones que distintos informantes observan de las conductas de niños y niñas en sus contextos naturales de desenvolvimiento. Los instrumentos de la batería ASEBA CBCL/1.5-5 y C-TRF son de amplia valoración en diversos países por su utilidad para estos propósitos. Estos instrumentos se gestaron a partir de una cuidadosa construcción psicométrica y su empleo, en diversos contextos, ha mostrado positivos indicadores de confiabilidad y validez de constructo, discriminante y predictiva.

Esta investigación ha tenido por objetivo evaluar el comportamiento de estos instrumentos en una muestra amplia y diversa socioeconómicamente, de preescolares chilenos, a fin de identificar si el uso de estos instrumentos en nuestro país, podría mostrar propiedades psicométricas análogas a las indicadas.

Los resultados obtenidos indican que en niñas y niños chilenos el modelo original de dos niveles tanto de la CBCL y C-TRF tiene un ajuste adecuado. El modelo de primer orden para ambos instrumentos, de 7 factores en para la CBCL/1.5-5, y de 6 para la C-TRF, ajustó bien. Como en la mayoría de los estudios, el ajuste de la CBCL fue más elevado que el de la C-TRF (Achenbach y Rescorla, 2000; Ivanova et al., 2010; Ivanova et al., 2011). El modelo bifactorial, que agrupa los factores de primer orden en dos dimensiones amplias de segundo orden, comportamientos internalizados y externalizados (y que deja fuera problemas del sueño), también mostró un ajuste adecuado. Sin embargo, para el logro de este ajuste se debió fijar la varianza residual del factor de reactividad emocional, de modo que fuese idéntica a la del factor ansiosodepresivo. Ambos factores se muestran muy interrelacionados y su carga relación a internalización, es sustantivamente más alta que la de aislamiento y quejas somáticas, lo que es importante de considerar al conceptualizar los comportamientos internalizados. La 
estrecha interrelación entre el factor reactividad emocional y ansiosodepresivo ha sido observada en estudios en diversas sociedades; sin embargo, la fusión de ambos factores no muestra siempre ser la mejor opción (Ivanova et al., 2007). Anteriores estudios, en muestras chilenas, habían mostrado adecuados ajustes del modelo original de la CBCL (Ivanova et al., 2010; Ivanova et al., 2011; Lecannelier et al., 2014) pero no así para la C-TRF (Ivanova et al., 2011).

Aun cuando se observó una consistencia interna de la mayoría de los factores de primer orden aceptable y comparable, aunque más baja en general, que la obtenida por los autores de los instrumentos, el uso más confiable de los instrumentos está dado por las escalas amplias de internalización, externalización y total. Debe destacarse sí que, al igual que en otros estudios en diversas sociedades, la correlación entre comportamientos internalizados y externalizados es importante, particularmente en la CBCL/1.5-5 (Liu et al., 2011).

En general, ambos instrumentos mostraron comportamientos análogos a los observados por los autores originales del instrumento y a los obtenidos en muestras transculturales, en particular en culturas "occidentales": escasos efectos de edad, con tendencia de algunos comportamientos a ser más señalados en niños y niñas de 2 ó 3 años que mayores, más identificación de comportamientos externalizados que internalizados; más identificación de comportamientos desadaptativos por parte de madres o padres que de educadores, más efectos de género en educadores que en madres o padres (Achenbachy Rescorla, 2000; Liu et al., 2011). Sin embargo, los efectos de género fueron más acentuados, observándose que tanto en madres o padres y educadores, informan más comportamientos externalizados y comportamientos desadaptativos globales en niños que niñas.

Un aspecto destacado a ser considerado es que en nuestro estudio los puntajes en la CBCL/1.5-5 fueron comparativamente altos. Ello no es atribuible a la sobre-representación en nuestro estudio del nivel socioeconómico bajo, dado que los valores se corresponden al estudio multicultural que agrupa a Chile entre las sociedades con más altos valores en este instrumento (Achenbachy Rescorla, 2010). Sin embargo, y también en convergencia con comparaciones multiculturales, en la C-TRF, los valores obtenidos se corresponden con los promedios de la mayoría de las sociedades estudiadas (Rescorla et al., 2012). A fin de facilitar el uso de ambos instrumentos en nuestro país, se han presentado, diferenciados por sexo, los valores de algunos centiles relevantes. Sin embargo, el valor clínico de los puntajes señalados no fue establecido. Achenbach y Rescorla (2000) identifican los valores sobre el percentil 83 y bajo el 90 como de "riesgo" y sobre 90 como de significación clínica, construyendo ese criterio sobre la capacidad de maximizar la capacidad de diferenciar entre niños y niñas derivados y no derivados a servicios de salud mental. Estos valores deben ser empleados, en consecuencia, como criterios referenciales solamente.

Los resultados obtenidos aportan antecedentes positivos para el empleo del CBCL/1.5 y C-TRF en nuestra población tanto en investigación, como para fines promocionales, preventivos o terapéuticos. Sin embargo, solo estudios longitudinales permitirán establecer la validez discriminante y predictiva de los puntajes en las diversas escalas.

\section{Referencias}

Achenbach, T. M., y Rescorla, L. (2000). Manual for the ASEBA preschool formsy profiles : an integrated system of multi-informant assessment. Burlington, VT: ASEBA.

Achenbach, T. M.,y Rescorla, L. (2010). Multicultural supplement to the Manual for the ASEBA Preschool Formsy Profiles. (Y. University of Vermont Research Center for Children, and Families. Ed.). Burlington, VT.

Egger, H. L., y Angold, A. (2006). Common emotional and behavioral disorders in preschool children: presentation, nosology, and epidemiology. Journal of Child Psychology and Psychiatry, 47, 313-337. doi: DOI 10.1111/j.1469-7610.2006.01618.x

Hofstra, M. B., van der Ende, J.,y Verhulst, F. C. (2002). Child and adolescent problems predict DSM-IV disorders in adulthood: A 14-year follow-up of a Dutch epidemiological sample. Journal of the American Academy of Child and Adolescent Psychiatry, 41, 182-189. doi: Doi 10.1097/00004583-200202000-00012

Ivanova, M. Y., Achenbach, T. M., Rescorla, L. A., Dumenci, L., Almqvist, F., Bilenberg, N., . . . Verhulst, F. C. (2007). The generalizability of the Youth Self-Report syndrome structure in 23 societies. Journal of Consulting and Clinical Psychology, 75, 729-738. doi: Doi 10.1037/0022006x.75.5.729

Ivanova, M. Y., Achenbach, T. M., Rescorla, L. A., Harder, V. S., Ang, R. P., Bilenberg, N., . . Verhulst, F. C. (2010). Preschool Psychopathology Reported by Parents in 23 Societies: Testing the Seven-Syndrome Model of the Child Behavior Checklist for Ages 1.5-5. Journal of the American Academy of Child and Adolescent Psychiatry, 49, 1215-1224. doi: 10.1016/j.jaac.2010.08.019

Ivanova, M. Y., Achenbach, T. M., Rescorla, L., Bilenberg, N., Bjarnadottir, G., Denner, S., . . Verhulst, F. (2011). Syndromes of Preschool Psychopathology Reported by Teachers and Caregivers in 14 Societies Using the Caregiver-Teacher Report Form (C-TRF). Journal of Early Childhoody Infant Psychology, 7, 87-103.

Lecannelier, F., Ewert, J. C. P., Groissman, S., Gallardo, D., Bardet, A. M., Bascunan, A.,y Rodriguez, J. (2014). Validation of Child Behavior Inventory for children ages $11 / 2$ to 5 years (CBCL $11 / 2-5$ ) at the Santiago de Chile City. Universitas Psychologica, 13, 491-500.

Liu, J. H., Cheng, H.,y Leung, P. W. L. (2011). The Application of the Preschool Child Behavior Checklist and the Caregiver-Teacher Report Form to Mainland Chinese Children: Syndrome Structure, Gender Differences, Country Effects, and Inter-Informant Agreement. Journal of Abnormal Child Psychology, 39, 251-264. doi: 10.1007/s10802010-9452-8

Mesman, J., Bongers, I. L.,y Koot, H. M. (2001). Preschool developmental pathways to preadolescent internalizing and externalizing problems. Journal of Child Psychology and Psychiatry and Allied Disciplines, 42, 679-689. doi: Doi 10.1017/S0021963001007351

Rescorla, L. A., Achenbach, T. M., Ivanova, M. Y., Bilenberg, N., Bjarnadottir, G., Denner, S., . . Verhulst, F. C. (2012). Behavioral/Emotional 
198 Félix Cova, Claudio Bustos, Paulina Rincón, Sandra Saldivia, Carolina Inostroza, Pamela Grandón

Problems of Preschoolers: Caregiver/Teacher Reports From 15 Societies. Journal of Emotional and Behavioral Disorders, 20, 68-81. doi: $10.1177 / 1063426611434158$

Rescorla, L. A., Bochicchio, L., Achenbach, T. M., Ivanova, M. Y., Almqvist, F., Begovac, I., . . . Verhulst, F. C. (2014). Parent-Teacher Agreement on Children's Problems in 21 Societies. Journal of Clinical Child and Adolescent Psychology, 43, 627-642. doi: 10.1080/15374416.2014.900719

Rosseel, Y. (2012). lavaan: An R Package for Structural Equation Modeling. Journal of Statistical Software, 48, 1-36.

Tan, T. X., Dedrick, R. F.,y Marfo, K. (2007). Factor structure and clinical implications of child behavior checklist/1.5-5 ratings in a sample of girls adopted from China. Journal of Pediatric Psychology, 32, 807-818. doi: 10.1093/jpepsy/jsm025 